\title{
Development and Performance Test of Deep Ultraviolet-Visible Photoacoustic Spectroscopy System for Nonradiative Deactivation Characterization in Phosphor Materials
}

\author{
Yutaka Fujimoto, ${ }^{1 *}$ Daisuke Nakauchi, ${ }^{2}$ Takayuki Yanagida, ${ }^{2}$ \\ Masanori Koshimizu, ${ }^{1}$ Haruki Fukada, ${ }^{3}$ Yamato Hayashi, ${ }^{1}$ and Keisuke Asai ${ }^{1}$ \\ ${ }^{1}$ Department of Applied Chemistry, Graduate School of Engineering, Tohoku University, Sendai 980-8579, Japan \\ ${ }^{2}$ Graduate School of Materials Science, Nara Institute of Science and Technology, \\ 8916-5 Takayama, Ikoma, Nara 630-0192, Japan \\ ${ }^{3}$ Electrical and Electronic Engineering, Kanazawa Institute of Technology, \\ 7-1 Ohgigaoka, Nonoichi, Ishikawa 921-8501, Japan
}

(Received December 19, 2019; accepted February 25, 2020)

Keywords: photoacoustic spectroscopy, nonradiative deactivation, scintillator, $\mathrm{Ce}: \mathrm{Y}_{3} \mathrm{Al}_{5} \mathrm{O}_{12}$

We developed a deep-ultraviolet-visible photoacoustic (PA) spectroscopy system for the evaluation of nonradiative deactivation in phosphor materials. The system consists of a xenon arc lamp of $300 \mathrm{~W}$ with an aspherical condensing mirror, a monochromator, a mechanical chopper to modulate the monochromatic light at a certain frequency of $10-100 \mathrm{~Hz}$, and a single optical fiber. The acoustic signal from the sample was counted with an electret microphone connected to a preamplifier, a sound level meter unit, and a lock-in amplifier. $0.25,0.5$, and $1.0 \mathrm{~mol} \% \mathrm{Ce}$-doped $\mathrm{Y}_{3} \mathrm{Al}_{5} \mathrm{O}_{12}$ crystalline samples were evaluated for the performance test of the system. From the evaluation, an inverse correlation among fluorescence quantum efficiency, scintillation light yield, and PA signal was experimentally confirmed.

\section{Introduction}

The photoacoustic (PA) effect was observed by Bell in the latter half of the 19th century, ${ }^{(1)}$ which is based on a heat generation process as a result of optical absorption in a sample. Almost sixty years later, Viengreov measured the concentration of particular gas in a mixture of gases using the PA effect, and after that, the technique has been applied in gas analysis. ${ }^{(2)}$ In 1976, Rosencwaig and Gersho reported their "Thermal Piston model", which is the theoretical treatment of the PA effect in solids. ${ }^{(3)}$ Photoacoustic spectroscopy (PAS) is a technique based on the PA effect, and it has attracted attention as a promising tool for measuring optical absorption spectra and for studying the nonradiative process in any type of medium, whether it be crystalline, powder or amorphous materials scattering strong light, and biological samples. ${ }^{(4-11)}$ Because of its advantage and merit, PAS has been widely used in the field of materials science. In the PAS technique, the PA signal is proportional to the optical absorbance of the sample and the probability of nonradiative transitions of the sample after excitation. ${ }^{(3)}$ Therefore, the

*Corresponding author: e-mail: fuji-you@qpc.che.tohoku.ac.jp https://doi.org/10.18494/SAM.2020.2746 
PAS technique can play an important role in understanding the luminescence properties and energy loss mechanisms of phosphor materials including scintillators for ionizing radiation detection. However, despite a large number of studies, most of them are based on only the characterizations of the radiative deactivation of the sample using luminescence spectra, decay time profiles, and temperature dependence, and other measurements. To the best of our knowledge, studies on luminescence dynamics and energy loss by experiments that directly evaluate the nonradiative deactivation are still few. ${ }^{(12-14)}$ For this reason, we developed a new PAS system that enables us to obtain information about nonradiative deactivation under modulated deep-ultraviolet (DUV)-visible (Vis) light excitation. The aim of this present work is to show the performance of the system by using Ce-doped yttrium aluminum garnet $\left(\mathrm{Y}_{3} \mathrm{Al}_{5} \mathrm{O}_{12}\right)$, which is a well-known phosphor for white $\mathrm{LED}^{(15,16)}$ and a scintillation detector. ${ }^{(17-19)}$ The Cedoped $\mathrm{Y}_{3} \mathrm{Al}_{5} \mathrm{O}_{12}$ crystal is the ideal sample to test the system we developed because of its high fluorescence quantum efficiency $(\mathrm{QE})$. Moreover, in this study, we experimentally examined the complementary relationships among fluorescence QE, scintillation light yield (LY), and PA signal using the crystals with different Ce concentrations.

\section{Methods}

Figure 1 shows a schematic diagram of the DUV-Vis PAS system (Bunkoukeiki). The excitation light source consists of a $300 \mathrm{~W}$ xenon arc lamp with an aspherical condensing mirror equipped with a monochromator (SM-5 High-Power Monochromator, Bunkoukeiki), a mechanical chopper (BCH-VL, Bunkoukeiki), and an optical fiber to modulate the monochromatic light. By using the optical fiber, we irradiated the modulated light onto the sample inside an original airtight PA cell through a quartz window. The PA cell is made of aluminum, and an electret microphone (UC-59, Rion) was mounted into the cell. The PA signal

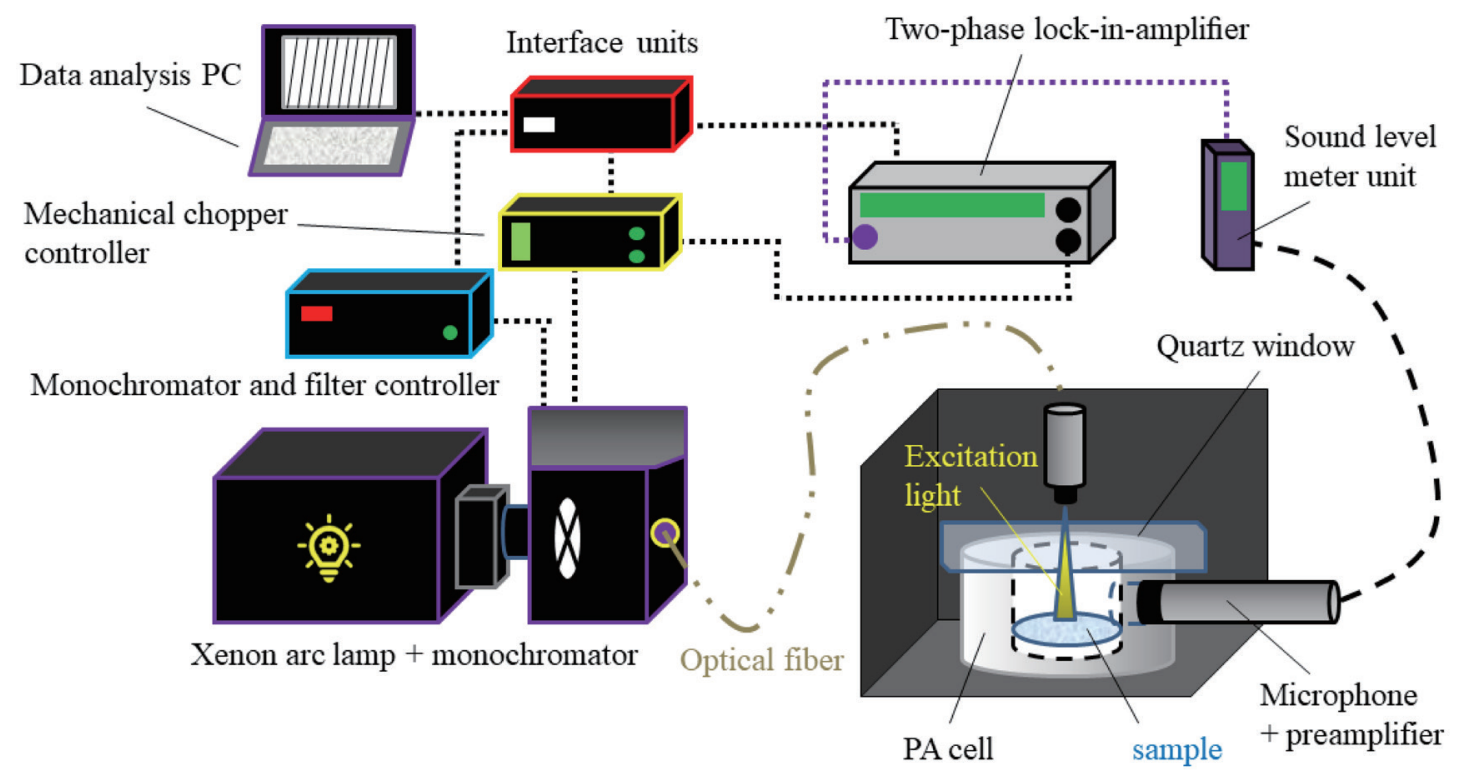

Fig. 1. (Color online) Schematic drawing of DUV-Vis PAS system. 
from the microphone was amplified by a preamplifier (NH-22A, Rion) and then fed into a sound level meter unit (UN-14, Rion) and a two-phase lock-in-amplifier (5610B, NF) with a reference signal input from the chopper. Finally, the signal from the lock-in-amplifier was converted into digital form and transmitted to a personal computer through an interface unit (STD-PRO2, Bunkoukeiki) for analysis. The obtained raw spectrum data are corrected for the wavelength dependence of both excitation light intensity using a carbon black reference and reflectance of the sample. Spectrum measurements were carried out at $2 \mathrm{~nm}$ steps in the wavelength range from 200 to $550 \mathrm{~nm}$ at a $19 \mathrm{~Hz}$ modulation frequency.

The diffuse reflectance spectra of the samples were measured with a V-700 UV-Vis spectrophotometer (Jasco) equipped with an integrating sphere for correction of the PA spectrum data. Crystal samples of the $0.25,0.5$, and $1.0 \mathrm{~mol} \%$ Ce-doped $\mathrm{Y}_{3} \mathrm{Al}_{5} \mathrm{O}_{12}$ were prepared by the floating zone method, as described in Refs. 20 and 21. The excitation spectra and fluorescence QE of the samples were evaluated with a F-7000 fluorescence spectrophotometer (Hitachi) and a Quantaurus-QY spectrofluorometer (Hamamatsu) equipped with a calibrated integrating sphere, ${ }^{(22,23)}$ respectively. To estimate the scintillation LY of the samples, ${ }^{137}$ Cs-gammaray-induced scintillation pulse height spectrum measurements were carried out by optically coupling the sample to a R7600U-20 photomultiplier tube (PMT, Hamamatsu). ${ }^{(24)}$ The setup for the measurements consisted of the PMT connected to a preamplifier (ORTEC 113), a shaping amplifier (ORTEC 572), and a multichannel amplifier (MCA8000D, Amptec). The obtained spectra were recorded with a shaping time of $3 \mu \mathrm{s}$. The scintillation LY of the samples was calculated by comparing the channel number at $662 \mathrm{kV}$ gamma-ray photopeak in the spectra with that of a CsI:Tl commercial scintillator $\left(L Y=\sim 50000\right.$ photons $\left./ \mathrm{MeV}, \lambda_{e m}=550 \mathrm{~nm}\right)$ under similar experimental conditions.

\section{Results and Discussion}

Figure 2(a) shows the PA spectra of the $0.25,0.5$, and 1.0 mol\% Ce-doped $\mathrm{Y}_{3} \mathrm{Al}_{5} \mathrm{O}_{12}$ crystals, and the excitation spectra are presented for comparison in Fig. 2(b). The PA spectra exhibited at least four bands at 230, 250-300, 335, and $460 \mathrm{~nm}$, whereas the excitation bands appeared at 230, 335, and $460 \mathrm{~nm}$. Similar PA bands were observed by Grinberg et al. ${ }^{(25)}$ By comparing with the excitation spectra, we found the PA bands at 230, 335, and $460 \mathrm{~nm}$ to be well consistent with the excitation bands of $\mathrm{Ce}^{3+}$. In general, the $\mathrm{Ce}^{3+}$ ion has the simplest electron configuration, and the $\mathrm{Ce}^{3+}$ emission results from electron transitions between the $4 \mathrm{f}$ ground state and the lowest $5 \mathrm{~d}^{1}$ excited state. The $4 \mathrm{f}$ ground state configuration splits into two sublevels, ${ }^{2} \mathrm{~F}_{5 / 2}$ and ${ }^{2} \mathrm{~F}_{7 / 2}$, which is caused by the spin-orbit interaction. On the other hand, the $5 \mathrm{~d}$ excited state configuration is split into at most five energetically different levels by the crystal field. Thus, the PA bands observed in the spectra correspond to the transitions from the $4 \mathrm{f}$ ground states to the $5 \mathrm{~d}^{3}(\sim 230 \mathrm{~nm}), 5 \mathrm{~d}^{2}(\sim 335 \mathrm{~nm})$, and $5 \mathrm{~d}^{1}(\sim 460 \mathrm{~nm})$ excited states of $\mathrm{Ce}^{3+}$. Results suggest that part of the excitation energy will be released as heat through the nonradiative deactivation process after excitation. The origin of the PA band at 250-320 nm cannot be understood clearly. However, we speculate that the origin is from strong absorption due to the F-type centers formed by oxygen vacancies in the host crystal. ${ }^{(26,27)}$ 


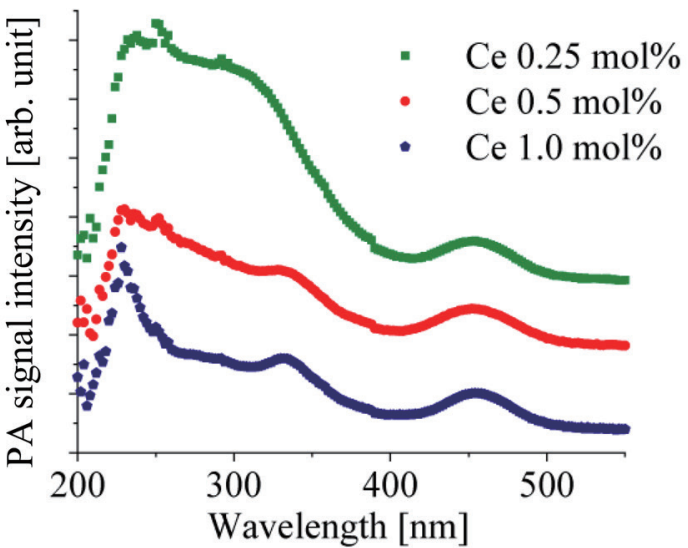

(a)

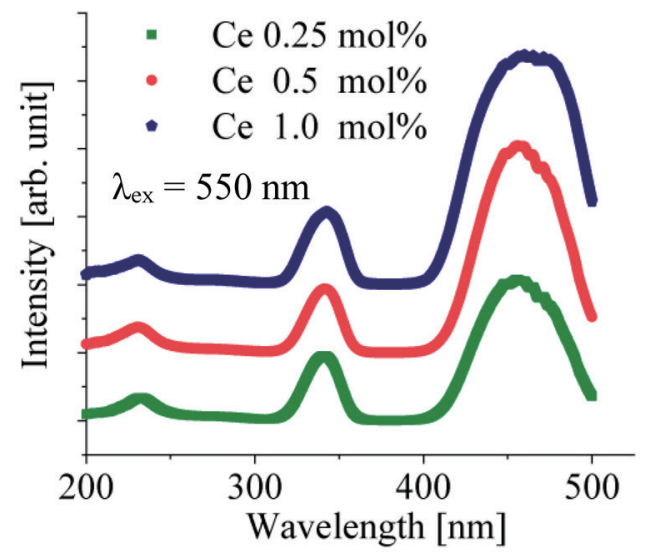

(b)

Fig. 2. (Color online) PA (a) and excitation (b) spectra of $0.25,0.5$, and $1.0 \mathrm{~mol} \%$ Ce-doped $\mathrm{Y}_{3} \mathrm{Al}_{5} \mathrm{O}_{12}$ crystals.

The excitation energy dependences of the PA signal and QE are shown in Figs. 3(a) and (b), respectively. The PA signal is determined according to the signal in the spectra under excitation at 335 and $460 \mathrm{~nm}$ light. From the calculation, the QEs of $\mathrm{Ce}^{3+}$ under excitation at 335 and $460 \mathrm{~nm}$ were approximately 63.8 and $86.8 \%$ for Ce $0.25 \mathrm{~mol} \%, 70.7$ and $93.4 \%$ for Ce $0.5 \mathrm{~mol} \%$, and 77.4 and $96 \%$ for Ce $1.0 \mathrm{~mol} \%$, respectively. QE and PA signal were found to exhibit a clear inverse correlation, which is in agreement with a complementary relationship from the viewpoint of conservation of energy. Besides, for all samples, the PA signal of the $\mathrm{Ce}^{3+}$ emission increased with the increase in excitation energy from $2.69 \mathrm{eV}(460 \mathrm{~nm})$ to $3.70 \mathrm{eV}$ $(335 \mathrm{~nm})$. The phenomena can be caused by some processes. One is the nonradiative transition between the high $5 \mathrm{~d}^{2}$ excited levels and the lowest $5 \mathrm{~d}^{1}$ excited level induced by phonons; the others are the photo and thermal ionization processes. ${ }^{(28,29)}$ From the measurements, the system we developed is found to be a very powerful tool for detecting and evaluating nonradiative deactivation with a high sensitivity because of the high QEs for the Ce-doped $\mathrm{Y}_{3} \mathrm{Al}_{5} \mathrm{O}_{12}$ crystals.

Figure 4 shows the Ce concentration dependences of the PA signal and QE under excitation at $460 \mathrm{~nm}$. As expected, the inverse correlation of PA signal with QE can also be observed. The PA signal gradually decreased with increasing Ce concentration, which may be caused by an improvement of the radiative deactivation rate according to an increase in QE. Additionally, no concentration quenching of the $\mathrm{Ce}^{3+}$ emission was observed in our prepared samples.

The scintillation pulse height spectra of the $0.25,0.5$, and $1.0 \mathrm{~mol} \%$ Ce-doped $\mathrm{Y}_{3} \mathrm{Al}_{5} \mathrm{O}_{12}$ crystals are shown in Fig. 5. The spectrum of the CsI:Tl commercial scintillator (black line) is presented for comparison. In the spectra, the ${ }^{137} \mathrm{Cs}-662 \mathrm{keV}$ gamma-ray photopeaks are located at about 400 channels for $\mathrm{Ce} 0.25 \mathrm{~mol} \%, 427$ channels for $\mathrm{Ce} 0.5 \mathrm{~mol} \%$, and 476 channels for Ce $1.0 \mathrm{~mol} \%$, whereas that of CsI:Tl was observed at 761 channels. Since the scintillation emission wavelength of the Ce-doped $\mathrm{Y}_{3} \mathrm{Al}_{5} \mathrm{O}_{12}(\sim 550 \mathrm{~nm})$ is close to that of the CsI:Tl scintillation $(\sim 550 \mathrm{~nm})$, they can be easily compared using the photopeak channel. Thus, the scintillation $\mathrm{LY}$ for $0.25,0.5$, and $1.0 \mathrm{~mol} \%$ Ce-doped $\mathrm{Y}_{3} \mathrm{Al}_{5} \mathrm{O}_{12}$ crystals can be estimated to be about 13100 , 14000 , and 15600 photons $/ \mathrm{MeV}$, respectively. 


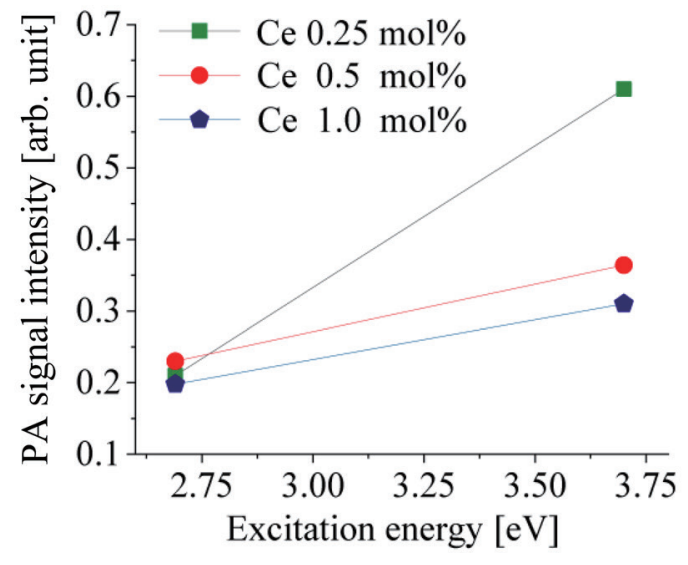

(a)

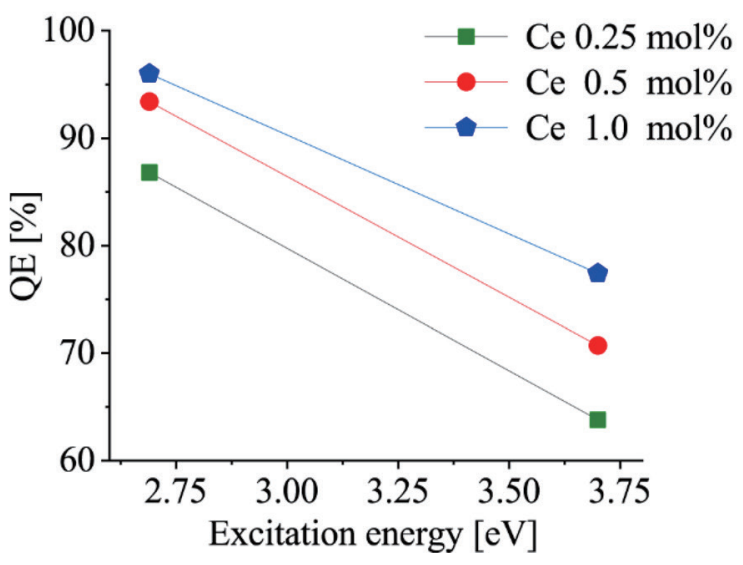

(b)

Fig. 3. (Color online) Excitation energy dependences of PA signal (a) and QE (b) of 0.25, 0.5, and $1.0 \mathrm{~mol} \% \mathrm{Ce}-$ doped $\mathrm{Y}_{3} \mathrm{Al}_{5} \mathrm{O}_{12}$ crystals.

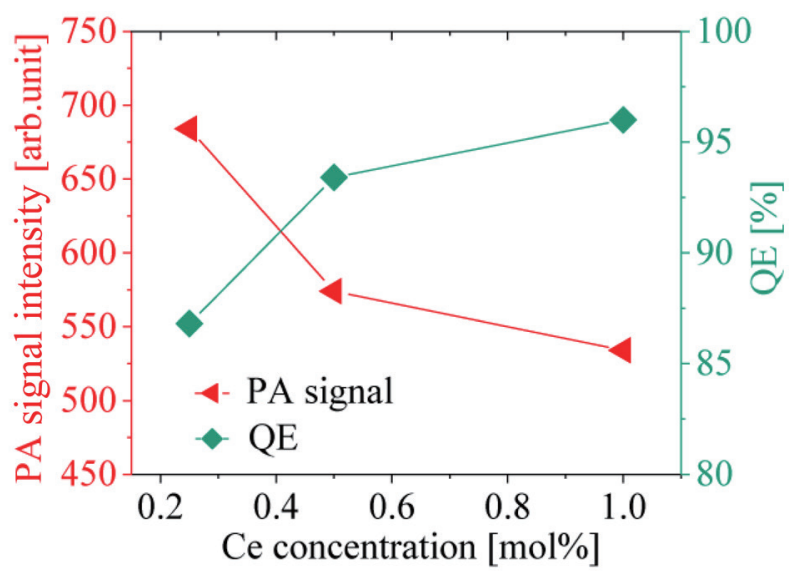

Fig. 4. (Color online) Variations of integrated PA signal and $\mathrm{QE}$ under excitation at $460 \mathrm{~nm}$ as function of Ce dopant concentration.

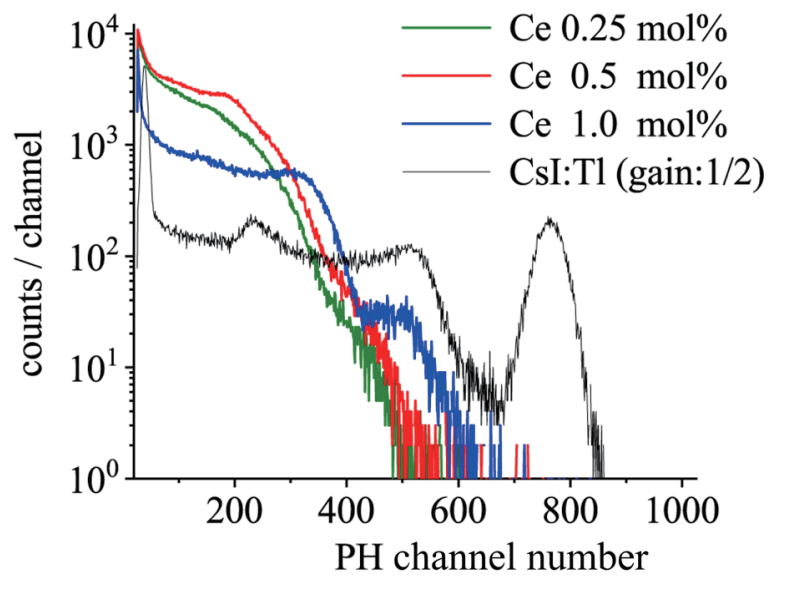

Fig. 5. (Color online) Scintillation pulse height spectra of $0.25,0.5$, and 1.0 mol\% Ce-doped $\mathrm{Y}_{3} \mathrm{Al}_{5} \mathrm{O}_{12}$ crystals in comparison with that of CsI:Tl scintillator.

Figure 6 shows the relationship between the integrated PA signal and the estimated scintillation LY at different Ce concentrations. The PA signal decreased with increasing scintillation LY. In other words, it exhibits an inverse correlation similarly to the relationship between $\mathrm{QE}$ and PA signal. Similar results were observed in $\mathrm{Cs}_{2} \mathrm{HfCl}_{6}: \mathrm{Te}^{4+}$ crystalline scintillators in an earlier study. ${ }^{(30)}$ To date, there are no theories to predict the performance of scintillators, and one phenomenological model (Robbins' model) is commonly used to explain the scintillation efficiency. ${ }^{(31,32)}$ The model is formulated as $L Y_{S C}=E /\left(\beta E_{g}\right) \times S \times Q$, where $L Y_{S C}$ is the scintillation LY, $E$ is the deposited energy of ionizing radiation, $\beta$ is the constant parameter, $E_{g}$ is the band gap energy, $S$ is the energy migration efficiency from the host to emission centers, and $Q$ is the fluorescence $\mathrm{QE}$ of the emission centers. The formula indicates that the scintillation LY is proportional to the fluorescence QE, which suggests that the 


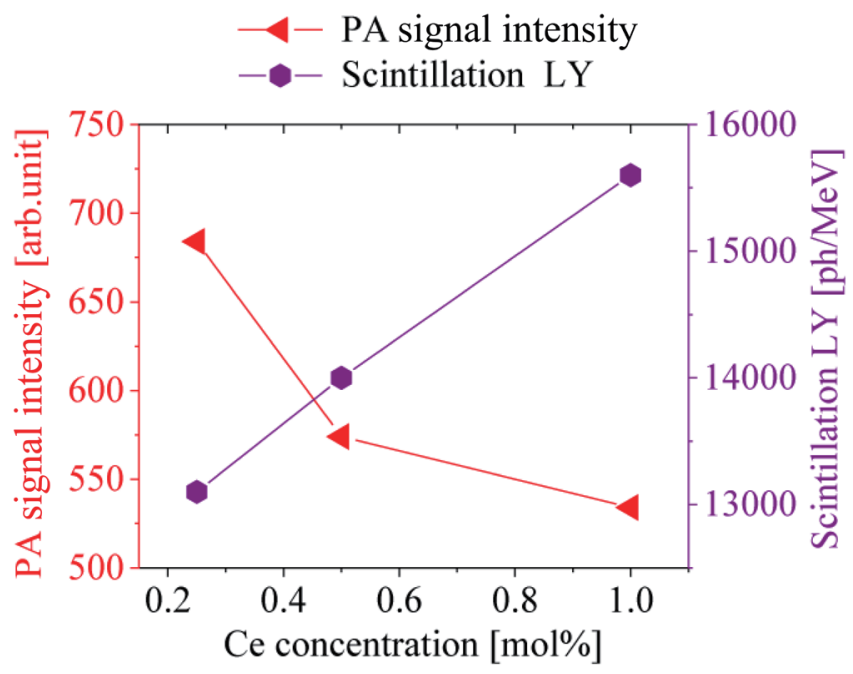

Fig. 6. (Color online) Variations of PA signal and scintillation LY as a function of Ce dopant concentration.

scintillation LY and PA signal show an inverse correlation because of the inverse relationship between QE (radiative deactivation) and PA signal (nonradiative deactivation) based on the energy conservation. Therefore, our experimental data obtained in this study are well consistent with Robbins' model, and are reasonable results based on the energy conservation.

\section{Summary}

In conclusion, we developed a DUV-Vis PAS evaluation system using a xenon arc lamp equipped with a monochromator and an electret microphone. $0.25,0.5$, and $1.0 \mathrm{~mol} \% \mathrm{Ce}$ doped $\mathrm{Y}_{3} \mathrm{Al}_{5} \mathrm{O}_{12}$ crystals were evaluated using the system, and it was confirmed that the system worked correctly with high sensitivity. The observed PA spectra were consistent with the excitation spectra and previous results, and our data suggest that the PA signal was inversely correlated with the fluorescence QE and scintillation LY.

\section{Acknowledgments}

This work was supported by the Cooperative Research Project of the Research Center for Biomedical Engineering, Konica Minolta Science and Technology Foundation, and Nakatani Foundation for Advancement of Measuring Technologies in Biomedical Engineering.

\section{References}

1 A. G. Bell: Am. J. Sci. 20 (1880) 305.

2 M. L. Viengreov: Dokl. Akad. Nauk. SSSR. 19 (1938) 687.

3 A. Rosencwaig and A. Gersho: J. Appl. Phys. 47 (1976) 64.

4 T. Ishiguro and H. Tokumoto: Jpn. J. Appl. Phys. 21 (1982) 11.

5 J. J. Freeman, R. M. Friedman, and H. S. Reichard: J. Phys. Chem. 84 (1980) 315.

6 A. Rosencwaig: Phys. Today 28 (1975) 23. 
7 A. Rosencwaig: Science 181 (1973) 657.

8 S. Yamasaki: Philos. Mag. B 56 (1987) 79.

9 G. Henderson and M. F. Bryant: Anal. Chem. 52 (1980) 1787.

10 D. Cahen, S. Malkin, and E. I. Lerner: FEBS Lett. 91 (1978) 339.

11 A. Rosencwaig and E. Pines: Biochim. Biophys. Acta 493 (1997) 10.

12 L. D. Merkle and R. C. Powell: Chem. Phys. Lett. 46 (1977) 303.

13 M. Morita: Jpn. J. Appl. Phys. 20 (1981) 296.

14 Z. J. Chen, J. W. Fang, and S. Y. Zhang: Int. J. Thermophys. 36 (2015) 947.

15 K. Bando, K. Sakano, Y. Noguchi, and Y. Shimizu: J. Light Vis. Env. 22 (1998) 2.

16 S. Nishiura, S. Tanabe, K. Fujioka, Y. Fujimoto, and M. Nakatsuka: IOP Conf. Ser. Mater. Sci. Eng. 1 (2009) 012031.

17 M. Moszynski, M. Kapusta, M. Mayhugh, D. Wolski, and S. O. Flyckt: IEEE Trans. Nucl. Sci. 44 (1997) 1052.

18 E. Zych, C. Brecher, A. J. Wojtowicz, and H. Lingertat: J. Lumin. 75 (1997) 193.

19 T. Yanagida, H. Takahashi, T. Ito, D. Kasama, T. Enoto, M. Sato, S. Hirakuri, M. Kokubun, K. Makishima, T. Yanagitani, H. Yagi, T. Shigeta, and T. Ito: IEEE Trans. Nucl. Sci. 52 (2005) 1836.

20 D. Nakauchi, G. Okada, N. Kawano, N. Kawaguchi, and T. Yanagida: Jpn. J. Appl. Phys. 57 (2018) 02 CB02.

21 M. Akatsuka, Y. Usui, D. Nakauchi, G. Okada, N. Kawaguchi, and T. Yanagida: Sens. Mater. 30 (2018) 1525.

22 K. Suzuki: Nat. Photon. 5 (2011) 247.

23 Y. Fujimoto, T. Yanagida, M. Koshimizu, and K. Asai: Sens. Mater. 27 (2015) 263.

24 Y. Fujimoto, K. Saeki, D. Nakauchi, T. Yanagida, M. Koshimizu, and K. Asai: Sens. Mater. 30 (2018) 1577.

25 M. Grinberg, A. Sikorska, and S. Kaczmarek: J. Alloy. Comp. 300-301 (2000) 158.

26 Y. Zorenko, T. Zorenko, T. Voznyakl, A. Mandowski, Q. Xia, M. Batentschuk, and J. Friedrich: IOP Conf. Series, Mater. Sci. Eng. 15 (2010) 012060.

27 Y. Fujimoto, T. Yanagida, H. Yagi, T. Yanagidani, and V. Chani: Opt. Mater. 36 (2014) 1926.

28 M. Koshimizu, T. Yanagida, K. Shinsho, S. Yanagisawa, Y. Fujimoto, H. Yagi, T. Yanagitani, and K. Asai: Nucl. Instrum. Methods Phys. Res., Sect. B 435 (2018) 285.

29 J. Ueda, J. Ceram. Soci. Jap. 123 (2015) 1059.

30 Y. Fujimoto, K. Saeki, D. Nakauchi, H. Fukada, T. Yanagida, H. Kawamoto, M. Koshimizu, and K. Asai: Mater. Res. Bull. 105 (2018) 291.

31 D. J. Robbins: J. Electrochem. Soc. 127 (1980) 2694.

32 T. Yanagida: Proc. Jpn. Acad. Ser. B 94 (2018) 75. 